This is an Open Access article distributed under the terms of the Creative Commons Attribution-Noncommercial License, which permits unrestricted use, distribution, and reproduction in any noncommercial medium, provided the original work is properly cited.

\title{
MID-INFRARED CAMERA W/WO LENS (MIRACLE) FOR SPICA
}

\section{Takehiko Wada, Hirokazu Kataza, and the SPICA pre-project team}

Japan Aerospace Exploration Agency, Institute of Space and Astronautical Science, 3-1-1 Yoshinodai, Sagamihara, Kanagawa 229-8510, Japan

\section{ABSTRACT}

Mid-InfRAred Camera w/wo LEns (MIRACLE) is the focal plane instrument designed for wide field imaging $\left(6^{\prime} \times 6^{\prime}\right)$ and low-resolution spectroscopic observations $(R=$ $100)$ over a wide spectral range in the mid-infrared wavelength $(5-38 \mu \mathrm{m})$. Thanks to the SPICA's cooled telescope, MIRACLE has a better sensitivity than JWST/MIRI at wavelengths over $20 \mu \mathrm{m}$ and its wider FOV provides a faster mapping speed in 5-26 $\mu \mathrm{m}$. MIRACLE opens a new wavelength region of $26-38 \mu \mathrm{m}$. Contiguous wavelength coverage is considered in the choice of the filter bands from the experiences in the Spitzer and AKARI observations. MIRACLE is equipped with a field stop wheel, which provides optimal slits in the spectroscopic mode and a wide FOV in the imaging mode. The results of a conceptual design study including sensitivity analysis are presented.

Key words: Instrumentation: infrared - Missions: SPICA

\section{INTRODUCTION}

As it is clearly demonstrated in the AKARI/IRC (Onaka et al., 2007) and Spitzer IRAC/MIPS (Fazio et al., 2004; Rieke et al., 2004) observations, mid-infrared wavelength is the key wavelength to study the physical conditions of the solid state interstellar medium and the evolution and formation of galaxy/star/planet in the dusty universe.

Multi-band imaging with contiguous wavelength coverage and low-resolution spectroscopy are important in this spectral range because broad emission and absorption lines of PAH and Silicate are dominant spectral features in this wavelength region, and useful to determine physical information by SED fitting techniques such as redshift and star formation rate (Figure 1).

A wide field of view is also critical for large area surveys and the study of variations of star formation activity in galaxies along the clustering scale (Figure 2). In order to make a merit of the state-of-the-art large format array detector, wide field of view is also desirable.

Mid-InfRAred Camera w/wo LEns (MIRACLE) is a focal plane instrument for SPICA (Nakagawa \& Murakami, 2007). MIRACLE is designed for wide field imaging and low-resolution spectroscopy with contiguous wavelength coverage in 5-38 $\mu \mathrm{m}$. The wavelength region of $26-38 \mu \mathrm{m}$ is the new window which MIRACLE explores.

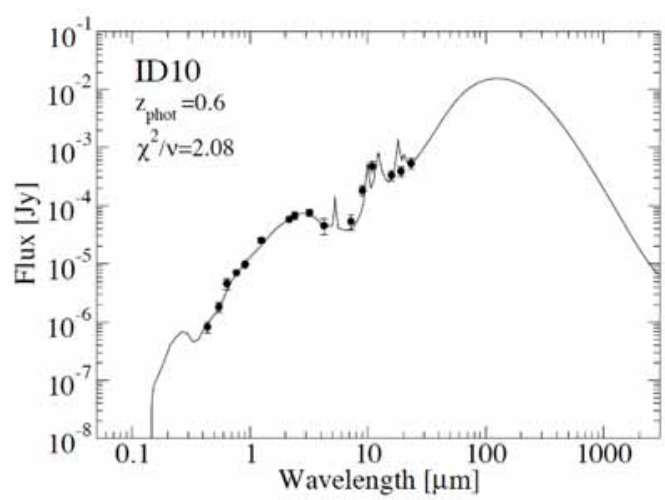

Figure 1. Example of MIR SED fitting for a galaxy obtained by AKARI/IRC MIR multi-band imaging observation. The SED is well fit by a dusty star forming galaxy at $z=0.6$. The board emission lines by PAH at the MIR provide a good diagnostic for redshift and star formation. (Takagi et al., 2007)

\section{SPECIFICATIONS}

Contiguous wavelength coverage over $5-38 \mu \mathrm{m}$ is required especially for tracing the $7.7 \mu \mathrm{m}$ PAH feature from galaxies in a wide range of redshift $(\mathrm{z}=0-4)$.

Wide field of view is required for faster mapping speed. One of the main targets of MIRACLE is to reveal the environmental effect of star forming activities around the distant clusters of galaxies. In order to achieve effective observations, a field of view covering a few arcmin. is required. At least, $4^{\prime} \times 4^{\prime}$ is required and $6^{\prime} \times 6^{\prime}$ is our goal (6' approximately correspond to co-moving transverse distance of $6 \mathrm{Mpc}$ at redshift $\mathrm{z}=1$ ).

In order to make a merit of the large aperture of the SPICA telescope, diffraction limited image quality must be achieved in the entire FOV.

Numbers of filters are required to cover contiguously for each channel with spectral resolution $\mathrm{R}=5-10$.

The specifications of MIRACLE are summarized in Table 1 . 


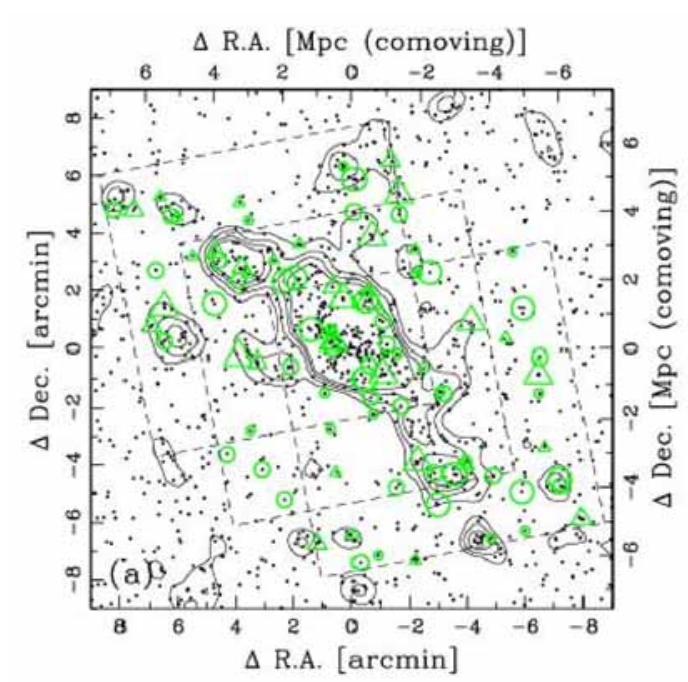

Figure 2. Example of MIR observation for distant cluster of galaxies $(z=0.81)$. Dots and contours are distribution and density of phot-z member galaxies. The dots marked with green triangles and circles are galaxies detected by AKARI/IRC $15 \mu \mathrm{m}$ survey and thought to be active star forming galaxies because the PAH emission at rest-frame $8 \mu \mathrm{m}$ comes into the $15 \mu \mathrm{m}$ band at this redshift. Clear enhancements of $15 \mu \mathrm{m}$ sources are found near over density regions, and the FOV of $6^{\prime} \times 6^{\prime}$ is useful to map out these galaxy clustering regions. (Koyama et al., 2008).

Table 1. Specification of MIRACLE.

\begin{tabular}{lcc}
\hline & Short channel & Long channel \\
\hline Wavelength & $5-26 \mu \mathrm{m}$ & $20-38 \mu \mathrm{m}$ \\
Spectral resolution & \multicolumn{2}{c}{$5-200$} \\
FOV & $4^{\prime} \times 4^{\prime}$ (min.) $-6^{\prime} \times 6^{\prime}$ (goal) \\
Image Quality & telescope diffraction limited \\
Noise & background limited (zodiacal light) \\
Observational mode & broad/narrow band imaging \\
& slit-less and slit spectroscopy \\
Number of filters & 6 (min.) -18 (goal) \\
Detector & Si:As 2Kx2K $\quad$ Si:Sb 1Kx1K \\
\hline
\end{tabular}

\section{PReliminary Design}

\subsection{OPTICAL DESIGN}

Figure 3 shows the conceptual design of the MIRACLE optics. The MIRACLE optics consists of two parts, foreoptics and rear-optics. Fore-optics is used as a rely optics and re-imaging the telescope focal plane. Filter wheels will be installed at the fore-optics pupil. In order to realize slit spectroscopy and wide-field imaging with a single instrument, we will install a field-mask wheel at the re-imaging focal plane. Dispersion elements will be installed at the rear-optics pupil. The detector will be installed at the final focal plane.

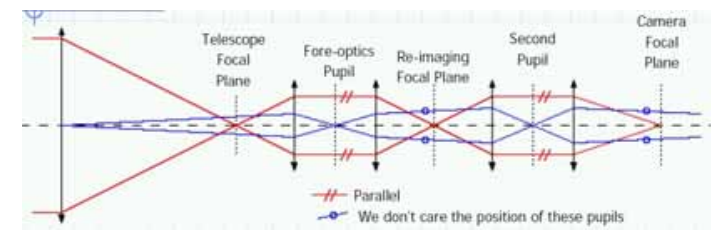

Figure 3. Design concept of MIRACLE optics.

Wide field optics without lens have been designed in order to cover wide wavelength range $(5-38 \mu \mathrm{m})$ with only two channels using confocal off-axis reflective imaging system (Figure 4 and Figure 6) based on the Chang's theory (Chang et al., 2006; Chang, 2008).

The current design has achieved diffraction limited performance (Strehl ratio is 0.9 or more) both for short (at $5 \mu \mathrm{m}$ ) and long (at $20 \mu \mathrm{m}$ ) channels in $6^{\prime} \times 6^{\prime}$ FOV (Figure 5 and Figure 7 ). The physical dimension is approximately $50 \times 100 \times 15 \mathrm{~cm}$ for each channel and meets the current system requirement. Optimization for volume and mass will be done subsequently.

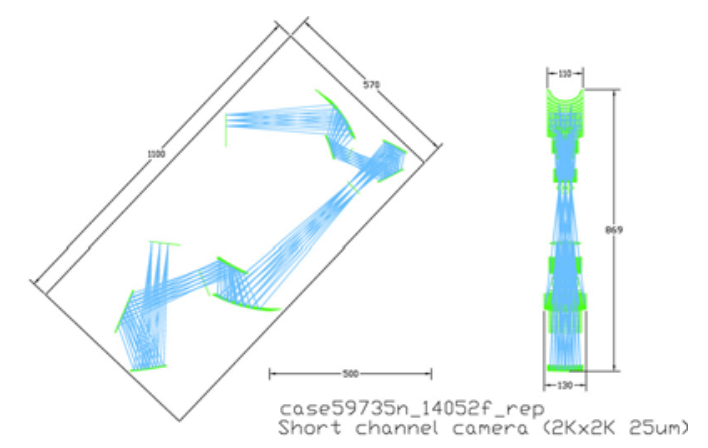

Figure 4. Ray-trace and configuration of MIRACLE short channel optics.

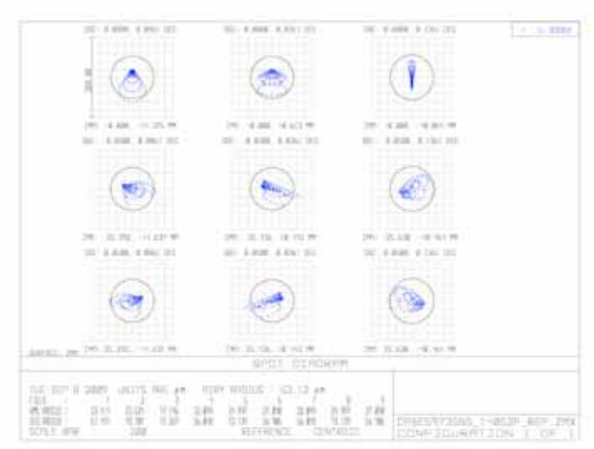

Figure 5. Spot diagram of MIRACLE short channel optics.

In order to achieve high efficiency in low resolution spectroscopic observations, we are planning to install a 


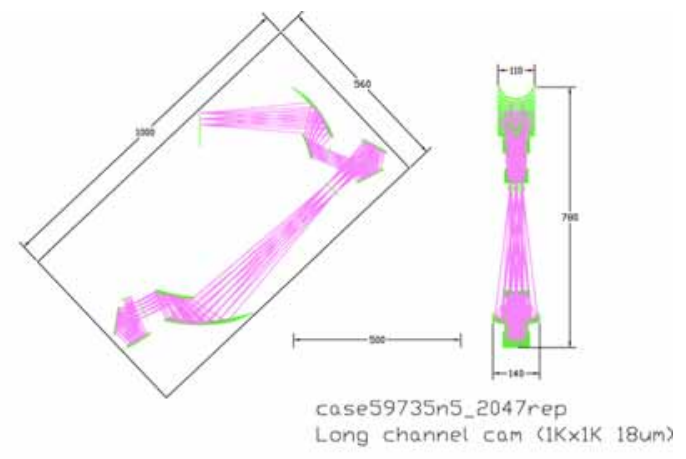

Figure 6. Ray-trace and configuration of MIRACLE long channel optics.

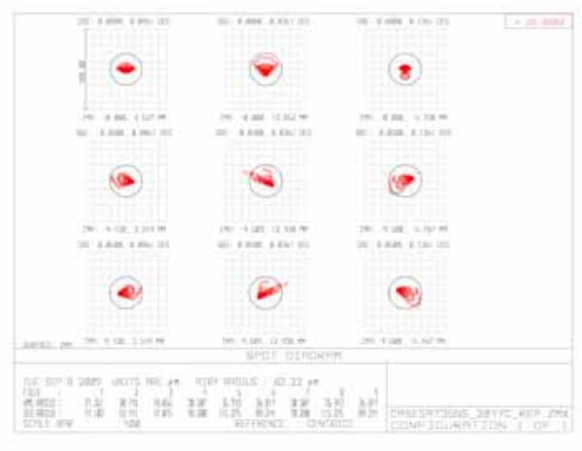

Figure \%. Spot diagram of MIRACLE long channel optics.

field-mask/slit wheel (Figure 9) at the focal plane of foreoptics. AKARI/IRC and JWST/MIRI used a fixed short slit at the edge of the field mask for low resolution slit spectroscopy. In case of AKARI/IRC, however, slit spectroscopic observations often suffer from electrical (MUX bleeding and/or column pull down) and optical (ghost) artificial effects cause by bright objects in the same field of view (Figure 8). In order to avoid these defects, a long-slit installed at the field-mask/slit wheel is used. A longer slit helps to maximize the observational efficiency for spectroscopic mapping observations.

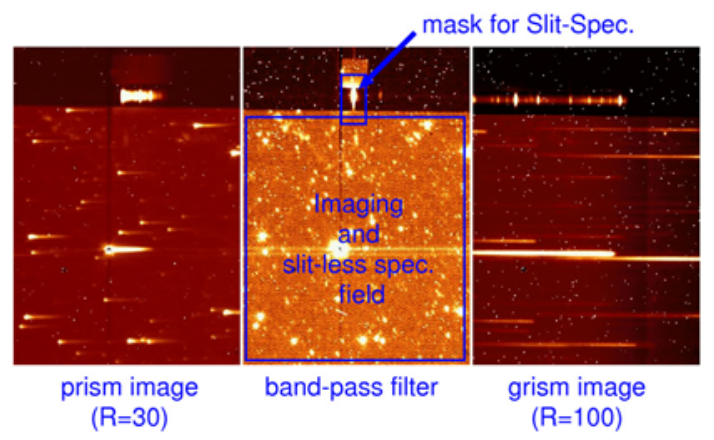

Figure 8. Example of slit-less and slit spectroscopy by AKARI/IRC.

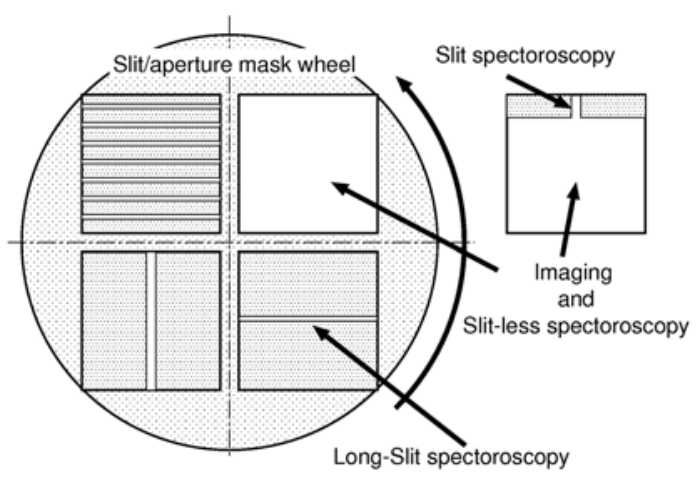

Figure 9. Schematic of field mask wheel.

\subsection{DETECTOR}

We are planed to use a $2 \mathrm{~K} \times 2 \mathrm{~K}$ Si:As IBC detector array for the short channel $(5-26 \mu \mathrm{m})$ and a $1 \mathrm{~K} \times 1 \mathrm{~K} \mathrm{Si:Sb} \mathrm{BIB}$ detector array for the long channel $(20-38 \mu \mathrm{m})$.

The $2 \mathrm{~K} \times 2 \mathrm{~K} \mathrm{Si}$ :As array is, however, in R\&D phase. If development of $2 \mathrm{~K} \times 2 \mathrm{~K} \mathrm{Si}$ As arrays is not ready in time, we will use existing $1 \mathrm{~K} \times 1 \mathrm{~K} \mathrm{Si}$ :As arrays. In this case, Nyquist sampling of the PSF is only achieved over $12 \mu \mathrm{m}$ in the short channel.

No larger than $1 \mathrm{~K} \times 1 \mathrm{~K}$ format is required for the long channel, because $1 \mathrm{~K} \times 1 \mathrm{~K}$ format detector already satisfies Nyquist sampling at $12 \mu \mathrm{m}$ even for the goal of FOV $\left(6^{\prime} \times 6^{\prime}\right)$.

\section{Sensitivity}

Sensitivity for point sources is calculated using the following assumptions.

- Read noise of 20 electron.

- Dark current of 1 electron/pixel.

- Pixel scale of $0.36 \mathrm{arcsec} / \mathrm{pixel}$.

- Optical efficiency of 0.35 including telescope.

- Detector efficiency of 0.50.

- Frame integration time of 617.3 seconds which is limited by cosmic ray event rate at the L2 environment.

- Total integration time of 3600 seconds.

- Aperture photometry with size of the first diffraction null ring.

Figure 10 shows the sensitivity for point sources compared with that of JWST/MIRI (Swinyard et al., 2004). MIRACLE is more sensitive compared to JWST/MIRI over wavelengths of $20 \mu \mathrm{m}$ because of SPICA's cooled aperture telescope.

Figure 11 shows the line sensitivity of MIRACLE in case of narrow-band imaging and slit spectroscopy.

Figure 13 shows the mapping speed of MIRACLE compared with that of JWST/MIRI (Swinyard et al., 2004). The wider FOV of MIRACLE provides a faster mapping speed in all JWST/MIRI bands. 


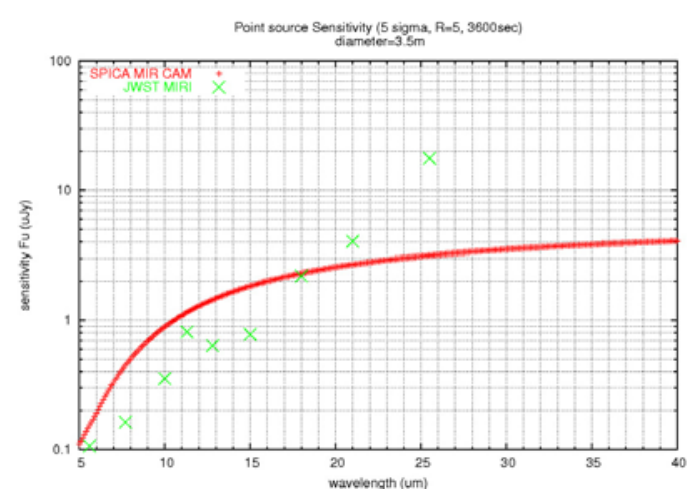

Figure 10. Sensitivity of MIRACLE. Red plus signs show point source sensitivity ( $5 \sigma$ ) of MIRACLE with one hour observing time in the imaging observations (spectral resolution $R=5$ ) as a function of wavelength. Green crosses show point source sensitivity of JWST/MIRI with the same integration. MIRACLE has superior sensitivity compared with JWST/MIRI over wavelengths of $20 \mu \mathrm{m}$ because of SPICA's cooled aperture telescope.

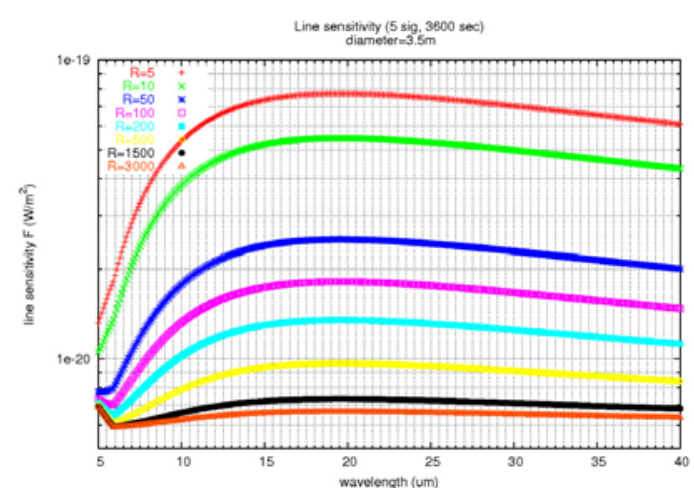

Figure 11. Line Sensitivity of MIRACLE spectroscopy. Calculation is based on narrow band imaging case. In case of slit-spectroscopy, the sensitivity is slightly worse. Results of medium resolution spectroscopy are just for reference.

\section{Summary}

Preliminary design of the SPICA MIR camera (MIRACLE for SPICA) is shown. MIRACLE opens a new wavelength window at $30 \mu \mathrm{m}$. MIRACLE has better sensitivity compared to JWST/MIRI over wavelength of $20 \mu \mathrm{m}$ and faster mapping speed in all MIRI bands. We have succeeded to design a reflective optical system with wide field of view. The current design meets the requirements such as contiguous wavelength coverage over $5-38 \mu \mathrm{m}$, wide field of view, diffraction limited image quality, natural background limited performance, and physical dimension. Optimization for mass is undergoing.

\section{REFERENCES}

Chang, S., Lee, J.H., Kim, S.P., et al. 2006, Applied Optics 45,484

Chang, S. 2008, COSPAR 37, 492

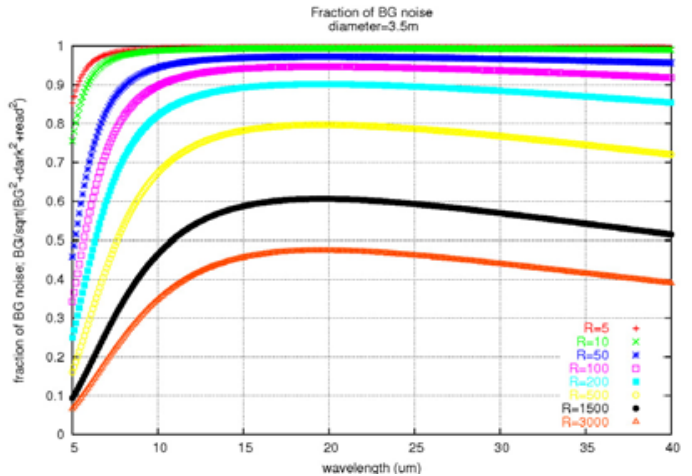

Figure 12. Contribution of natural background photon noise to total noise as a function of wavelength is shown. Natural background (Zodiacal light) photon noise limited observations are achieved in wavelengths over $10 \mu \mathrm{m}$ for low resolution spectroscopy. Read noise is dominant at wavelengths close to $5 \mu \mathrm{m}$.

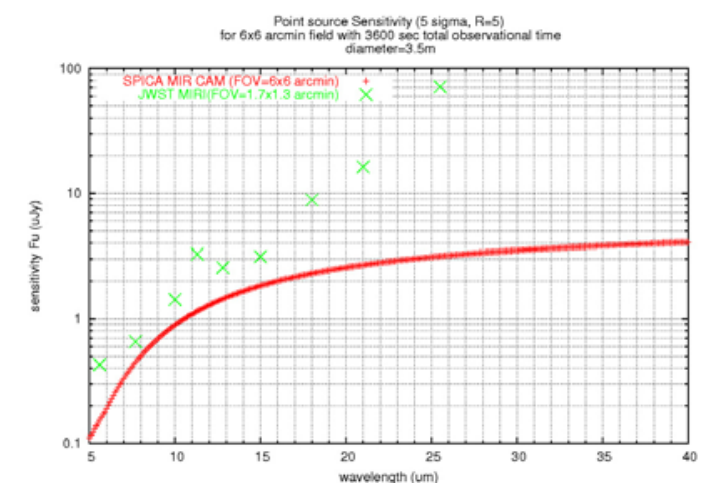

Figure 13. Mapping speed of MIRACLE. Red plus signs show point source sensitivity ( $5 \sigma$ ) of MIRACLE with one hour observing time in the imaging observations (spectral resolution $R=5$ ) as a function of wavelengths. Green crosses show point source sensitivity of JWST/MIRI survey covering $6^{\prime} \times 6^{\prime}$ field with the same observing time. MIRACLE has superior mapping speed compared with JWST/MIRI over the entire MIRI wavelength coverage.

Fazio, G.G., Hora, J.L., Allen, L.E., et al. 2004, ApJS, 154,10

Koyama, Y., Kodama, T., Shimasaku, K., et al. 2008, MNRAS 391, 1758

Nakagawa, T., \& Murakami, H. 2007, AdSpR 40, 679

Onaka, T., Matsuhara, H., Wada, T., et al. 2007, PASJ 59, S401

Rieke, G.H., Young, E.T., Engelbracht, C.W., et al. 2004, ApJS, 154, 25

Takagi, T., Vansevicius, V., Arimoto, N., et al. 2003, PASJ 55,385

Takagi, T., Hanami, H., Arimoto, N., et al. 2004, MNRAS 355,424

Takagi, T., Matsuhara, H., Wada, T., et al. 2007, PASJ $59, \mathrm{~S} 557$ 


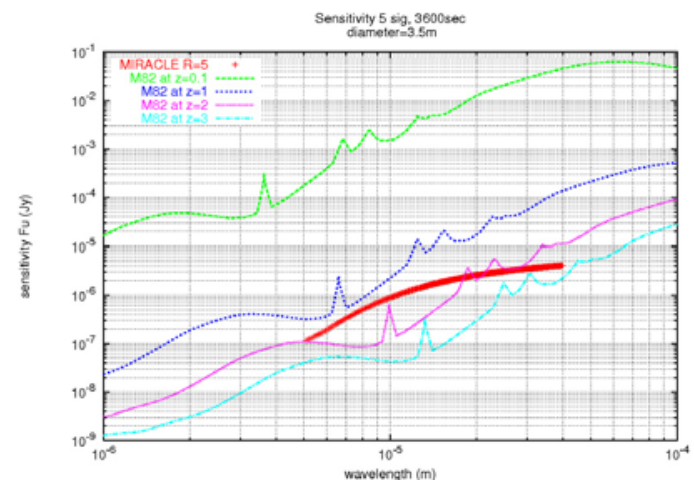

Figure 14. Detectability of the low luminosity star-burst galaxy (M82) at a variety of redshifts by MIRACLE is demonstrated. The SED is taken from the SED models of StarBUrst with Radiative Transfer (SBURT) by Takagi et al. (2003, 2004) adopting the LMC type extinction curve, age of 0.03 Gyr and optical depth $\tau_{V}=9.160$, and scaled to total luminosity of $3.7 \times 10^{10} \mathrm{~L} \odot$. Rest frame $7.7 \mu \mathrm{m}$ PAH feature is detectable at redshift $z=2$.

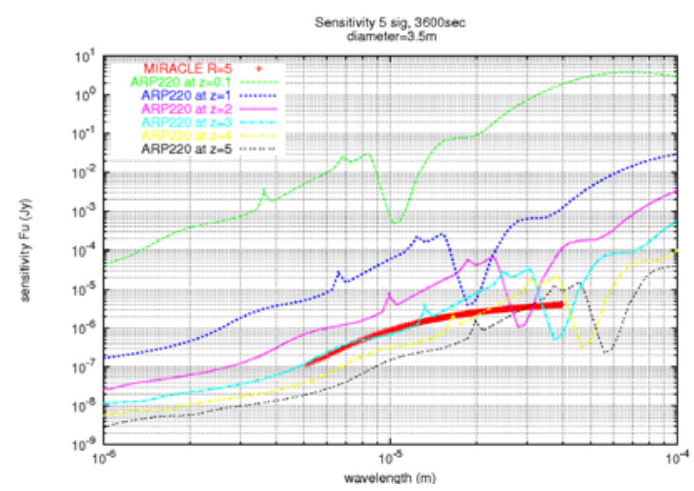

Figure 15. Detectability of ULIRG (Arp220) at a variety of redshifts by MIRACLE is demonstrated. The SED is taken from the SED models of StarBUrst with Radiative Transfer (SBURT) by Takagi et al. (2003, 2004) adopting the SMC type extinction curve, age of 0.03 Gyr and optical depth $\tau_{V}=43.670$, and scaled to total luminosity of $1.4 \times 10^{12} L_{\odot}$. Rest frame $7.7 \mu \mathrm{m}$ PAH feature is detectable at redshift $z=4$.

Swinyard, B.M., Rieke, G.H., Ressler, M., et al. 2004, SPIE 5487, 785 